\title{
Nutritional Treatment: New Strategy for Management of Chronic Pelvic Pain
}

\author{
Jong Soon Choi ${ }^{1}$, Heung Yeol Kim ${ }^{2}$ \\ ${ }^{1}$ Department of Family Medicine, College of Medicine, Kosin University, Busan, Korea \\ ${ }^{2}$ Department of Obstetrics and Gynecology, College of Medicine, Kosin University, Busan, Korea
}

Chronic pelvic pain is a common condition in women of reproductive age and can be described as chronic nociceptive, inflammatory and neuropathic pain characterized by spontaneous pain or a response to various stimuli. Oxidative stress is a component of the inflammatory reaction associated with pain processes. Iron and NF- $\kappa \mathrm{B}$ are well-known inducers of oxidative stress, and reactive oxygen species (ROS) are associated with chronic pelvic pain and play an important role in the regulation of genes expressing immunoregulators, cytokines, and other molecules. Chronic pelvic pain treatment is often unsatisfactory and limited to symptom control. However, dietary treatment with antioxidants can improve the function of the immune system and overcome free radical damage. Therefore, dietary supplementation is suggested as a means to treat some chronic medical conditions that respond poorly to medication. In summary, dietary treatment with antioxidants could be considered for new strategy for treatment of chronic pelvic pain and may be better tolerated by patients than current treatments.

Key Words: Antioxidant, Chronic pelvic pain, Inflammation, Oxidative stress

Chronic pelvic pain (CPP) is a common condition in women of reproductive age; rates of consultation for CPP in general practice are similar to those for asthma and migraine. ${ }^{1}$ CPP can be identified as a constellation of chronic inflammatory processes from various stimuli, such as oxidative stress, toxins, and chemicals. To treat CPP, a multidisciplinary approach to care and management produces the best results. ${ }^{2}$

Hydroxyl radicals are the most reactive free radical species known. They have the ability to react with a wide range of cellular constituents such as amino acid residues and purine and pyrimidine DNA bases to initiate a free radical chain reaction. Oxidative stress has been proposed as a potential factor that may be involved in endometriosis, ${ }^{3}$ a chronic pelvic inflammatory process. Two inflammatory mediators thought to be involved in $\mathrm{CPP}$ are iron and $\mathrm{NF}-\mathrm{kB}$.

The purpose of this article was to show that dietary treatment with antioxidants improves the function of the immune system and fights free radical damage. Agents with antioxidant activity may relieve CPP without side effects. Therefore, using nutrition treatments such as dietary antioxidants (magnesium, vitamin D) may prove beneficial for CPP control.

\section{Definition}

$\mathrm{CPP}$ is a common disease in women of reproductive age; it causes disability and distress and also results in significant costs to health services. ${ }^{4}$ The most commonly used definition of CPP considers only the
Corresponding Author: Heung Yeol Kim, Department of Obstetrics and Gynecology, College of Medicine, Kosin University, 34 Amnamdong, Seo-gu, Busan, 602-702, Korea

TEL: +82-51-990-6226 FAX: +82-51-990-3300 E-mail: hykyale@yahoo.com
Received: July 18, 2012

Revised: July 22, 2012

Accepted: August 17, 2012 
location and duration of the pain: recurrent or constant pain in the lower abdominal region that has lasted for at least six months.

CPP can be identified as a chronic nociceptive, inflammatory and neuropathic pain characterized by spontaneous appearance and a response to painful, innocuous stimuli. ${ }^{5}$

CPP is a diagnostic and management challenge that requires a multisystem approach. This condition may have a single cause or multiple causes, and some women never receive a definitive diagnosis. CPP is also associated with psychological conditions however, the establishment of psychosocial factors as cause or effect of the pain is difficult.

\section{Current treatment approach of CPP}

According to one study, the current treatment approach is counseling supported by ultrasound monitoring, psychotherapy, laparoscopy to exclude serious pelvic pathology, hormonal therapy, and neuroablative treatment to interrupt nerve pathways. ${ }^{5}$ Another report indicated that management includes counseling or psychotherapy, attempting to provide reassurance using laparoscopy to exclude serious pathology, progestogen therapy (such as medroxyprogesterone acetate), and surgery to interrupt nerve pathways. $^{4}$

Laparoscopic surgery and treatment with medroxyprogesterone acetate, danazol or nafarelin were more effective than control treatments. Medical therapy after surgical treatment significantly reduced pain, but six months after therapy was stopped there was no difference between women who were treated and those who were not treated postoperatively. ${ }^{6}$

Because CPP treatment is often unsatisfactory and limited to partial symptom relief, we suggest dietary supplementation as a means to treat chronic medical illnesses that respond poorly to prescription medications. Dietary therapy with antioxidants improves the function of the immune system and fights free radical damage. Another advantage is that antioxidants can improve CPP without side effects.

\section{Effect of oxidative stress}

Hydroxyl radicals are the most reactive free radical species known and have the ability to react with a wide range of cellular constituents, such as amino acid residues and purine and pyrimidine DNA bases to initiate a free radical chain reaction known as lipid peroxidation. Oxidative stress has been proposed as a potential factor involved in endometriosis. ${ }^{3}$

ROS are intermediate species produced by normal oxygen metabolism. To protect themselves from ROS, cells have developed a wide range of antioxidant systems to inactivate ROS, limit ROS production, and repair cell damage.

Univalent reduction of oxygen results in production of the superoxide anion. The dismutation of two superoxide anions, catalyzed by superoxide dismutase, leads to the formation of hydrogen peroxide. Hydrogen peroxide is detoxified by glutathione peroxidase or inactivated to $\mathrm{H} 20$ and $\mathrm{O} 2$. Optimal protection is achieved only when an appropriated balance among superoxide dismutase, glutathione peroxidase, and catalase is maintained. The hydroxyl radical is highly toxic to cells asit can react with all cellular components.

The body's antioxidative system includes enzymatic antioxidants such as catalase, SOD, glutathion peroxidase, thioredoxin reductase, G. reductase, G.Stransferase, and G6PD. Nonenzymatic antioxidants are known as endogenous antioxidants (glutathion- 
$\mathrm{SH}$, cysteine-SH, bilirubin, uric acid, lipoic acid, coenzyme Q10) while exogenous antioxidants include vitamins $\mathrm{C}$ and $\mathrm{E}$, beta-carotene, lycopene, selenium, copper, zinc, and magnesium.

Oxidative stress may occur when the balance between ROS production and antioxidant defense is disrupted. The increasing number of diseases associated with oxidative stress suggests that the oxidative balance may be precarious. ${ }^{7}$ Overexpression of ROS induces cellular damage and impairs cellular function by altering protein activity and gene expression. ROS are important in regulating redox-sensitive transcription factors implicated in endometriosis. ${ }^{8}$ Oxidative stress occurs in endometriosis via expression of xanthine oxidase, an enzyme that produces ROS. ${ }^{9}$

Two inflammatory mediators have been identified: iron and $\mathrm{NF}-\mathrm{kB}$. Iron can generate free radical species that are able to react with a wide range of cellular constituents, thus inducing cellular damage. Overproduction of ROS impairs cellular function by altering gene expression via regulation of redox-sensitive transcription factors such as NF-kB. Iron overload provokes iron-mediated damage, oxidative injury, and inflammation. ${ }^{10}$ Iron is known to induce oxidative stress, leading to macromolecular oxidative damage, tissue injury and chronic inflammation. Iron was recently suggested to be involved in endometriosis development. Oxidative stress and proinflammatory cytokines are potent activators of the NF-kB pathway. ${ }^{11} \mathrm{NF}-\mathrm{kB}$ activation leads to expression of proinflammatory genes like cytokines, which may provide positive feedback to the pathway. Inflammatory cytokines (interleukin-1,6, TNF-alpha) are increased and activated in endometriosis patients. ${ }^{12}$ $\mathrm{NF}-\mathrm{kB}$ is activated by diverse proinflammatory stimuli such as tumor necrosis factor (TNF)-alpha,
LPS, and oxidative stress, and may lead to multiple genes implicated in inflammation, immunity, apoptosis, cell proliferation, tissue invasion and angiogenesis.

\section{Vitamin $D$ and pelvic pain}

Vitamin D is a lipid-soluble micronutrient produced in the skin when provitamin $\mathrm{D}$ (7-dehydrocholesterol) in cell membranes is exposed to ultraviolet $B$ rays and converted into cholecalciferol (D3). Circulating D3 is then bound by vitamin D binding protein and transported in serum to be stored in adipose tissue or delivered to the liver, where it is converted to 25-hydroxyvitamin D2 [25 (OH)D]. This metabolite is referred to as the total $25(\mathrm{OH}) \mathrm{D}$ and is measured in most clinical studies. The 25-hydroxyvitamin D2 is then activated by conversion to calcitriol in the kidney. Synthesis of $25(\mathrm{OH}) \mathrm{D}$ and 1,25 $(\mathrm{OH}) 2 \mathrm{D} 3$ is coupled with calcium homeostasis. Serum levels of vitamin $\mathrm{D}$ are regulated by parathyroid hormone, phosphorus, and calcium levels.

Vitamin $\mathrm{D}$ is a micronutrient vital in calcium homeostasis levels and musculoskeletal function. Vitamin D insufficiency shows clinical signs of rickets and osteomalacia. The pelvic floor is a unique part of the body, and its function is dependent on interrelationships between muscle, nerve, connective tissue, and bone. Pelvic floor disorders result when these relationships are disrupted.

Pelvic floor muscle weakness is clinically observed in women with pelvic floor disorder symptoms and may be impacted by insufficient serum vitamin $\mathrm{D}$ levels. Sufficient vitamin D serum levels may facilitate an increase in pelvic floor muscle efficiency and a decrease in detrusor contractibility. Vitamin D regulates inflammatory cytokine levels, ${ }^{13}$ and calci- 
triol also stimulates antioxidant gene expression in a dose-response manner, making it a potential target as a pharmacologic pro-oxidant. ${ }^{14}$

Vitamin E levels are also significantly lower in the peritoneal fluid of women with endometriosis because antioxidants in peritoneal fluid are consumed during oxidation reactions. Vitamin E plays an important role in protecting biological membranes by preventing peroxidation. It may also help prevent activation of redox-sensitive pathways, which have been implicated in abnormal cell proliferation and inflammatory responses. $^{15}$

Free radical scavengers, such as vitamin $\mathrm{C}$ and $\mathrm{E}$, neutralize free radicals in chronic inflammatory disease.

\section{Magnesium and pelvic pain}

Magnesium was recognized as an essential nutrient in mice in $1932,{ }^{16}$ and was also accepted as an important nutrient in humans. ${ }^{17}$ Magnesium deficiency is not uncommon among the general population. Its intake has decreased over the years, especially in the Western world. ${ }^{18}$ Low magnesium status has been associated with numerous pathological conditions characterized as having a chronic inflammatory stress component. Recently, magnesium deficiency was suggested as a risk factor in some chronic diseases, such as osteoporosis, cardiovascular disease, and diabetes. Magnesium deficiency is now considered to contribute to chronic diseases, and the role of magnesium as a therapeutic agent is being tested in numerous large clinical trials. For example, recent research showed that lower serum magnesium $(\mathrm{Mg})$ levels are associated with chronic kidney disease. ${ }^{19}$

Hypomagnesemia was reported to promote endothelial cell dysfunction, leading to inflammation and oxidative stress. ${ }^{20}$ Magnesium sulfate administration inhibits placental inflammation during LPS-mediated maternal infection and attenuates excessive inflammation at the maternal-fetal interface. Several placental inflammatory genes which are regulated by LPS were silenced by magnesium sulfate treatment. ${ }^{21}$ In rats, magnesium deficiency over only a few days induced a clinical inflammatory syndrome characterized by macrophage activation, release of inflammatory cytokines and acute phase proteins, and excessive production of free radicals. When magnesium concentration was increased, the inflammatory response decreased. Thus, reduction in the extracellular magnesium resulted in cell activation. ${ }^{22}$

Therefore, since magnesium reduces inflammatory syndromes and inhibits oxidative stress. magnesium supplementation can be beneficial in treatment of chronic diseases such as chronic pelvic pain.

\section{Vitamin $E$ and pelvic pain}

Vitamin E levels are significantly lower in the women with endometriosis because anti-oxidants consumed during oxidataion reactions. ${ }^{23,24}$ Patients with endometriosis not only presented increased lipid peroxidation but also maintained lower vitamin $\mathrm{E}$ levels than the control group. ${ }^{25}$ Vitamins $\mathrm{C}$ and $\mathrm{E}$ supplementation was associated with a decrease in the concentration of oxidative stress markers in women with endometriosis. ${ }^{26}$

Vitamin $\mathrm{E}$ have important action in protecting biological membranes by preventing peroxidation. It may also play a role in preventing activation of redox-sensitive pathways, which implicated in abnormal cell proliferation and inflammatory response. 


\section{CONCLUSION}

CPP can be identified as a group of chronic inflammatory processes resulting from various stimuli, such as oxidative stress, toxins, or chemicals. Current treatment approaches include counseling supported by ultrasound monitoring, psychotherapy, and laparoscopy to exclude serious pelvic conditions. Recently, dietary therapy studies have suggested that antioxidants improve the function of the immune system and fight free radical damage. Vitamin D in particular regulates inflammatory cytokine levels calcitriol also stimulates antioxidant gene expression because pelvic floor disorder symptoms may be impacted by insufficient serum vitamin D.

Hypomagnesemia was reported to induce endothelial cell dysfunction and lead to inflammation and oxidative stress. Therefore, magnesium supplementation can be beneficial in chronic diseases, such as CPP. In conclusion, dietary therapy with antioxidants could be considered as a new effective strategy in the long-term management of chronic pelvic pain.

\section{REFERENCE}

1. Cheong Y, William Stones R. Chronic pelvic pain: aetiology and therapy. Best Pract Res Clin Obstet Gynaecol 2006;20: 695-711.

2. Gelbaya TA, El-Halwagy HE. Focus on primary care: chronic pelvic pain in women. Obstet Gynecol Surv 2001;56:757-64.

3. Gupta S, Agarwal A, Sekhon L, Krajcir N, Cocuzza M, Falcone T. Serum and peritoneal abnormalities in endometriosis: potential use as diagnostic markers. Minerva Ginecol 2006;58: 527-51.

4. Stones RW, Mountfield J. Interventions for treating chronic pelvic pain in women. Cochrane Database Syst Rev 2000;4: CD000387.

5. Sesti F, Capozzolo T, Pietropolli A, Collalti M, Bollea MR, Piccione E. Dietary therapy: a new wtrategy for management of chronic pelvic pain. Nutr Res Rev 2010;25:1-8.

6. Howard FM. An evidence-based medicine approach to the treatment of endometriosis-associated chronic pelvic pain: placebo-controlled studies. J Am Assoc Gynecol Laparosc 2000;7:477-88.

7. Hippeli S, Elstner EF. Transitional metal ion-catalyzed oxygen activation during pathogenic processes. FEBS Lett 1999;443: $1-7$.

8. Guo SW, Nuclear factor-kappa b (NF-kB): an unsuspected major culprit in the pathogenesis of endometriosis that is still at large? Gynecol Obstet Invest 2007;63:71-97.

9. Ota H, Igarashi S, Tnaka T. Xanthine oxidase in eutopic and ectopic endometrium in endometriosis and adenomyosis. Fertil Steril 2001;75:785-90.

10. Xi G, Keep RF, Hoff JT. Mechanisms of brain injury after intracerebral haemorrhage. Lancet Neurol 2006:5:53-63.

11. Lousse JC, Van Langendonckt A, González-Ramos R, Defrère $\mathrm{S}$, Renkin E, Donnez J. Increased activation of nuclear factor-kappa B (NF-kappaB) in isolated peritoneal macrophages of patients with endometriosis. Fertil Steril 2008;90:217-20.

12. Montagna P, Capellino S, Villaggio B, Remorgida V, Ragni $\mathrm{N}$, Cutolo $\mathrm{M}$, et al. Peritoneal fluid macrophages in endometriosis: correalation between the expression of estrogen receptors and inflammation. Fertil Steril 2008;90:156-64.

13. Liu NQ, Kaplan AT, Lagishetty V, Ouyang YB, Ouyang Y, Simmons CF, et al. Vitamin D and the regylation of placental inflammation. J Immunol 2011;186:5968-74.

14. Halhali A, Figueras AG, Díaz L, Avila E, Barrera D, Hernández $\mathrm{G}$, et al. Effects of calcitriol on calbindins gene expression and lipid peroxidation in human placenta. J Steroid Biochem Mol Biol 2010;121:448-51.

15. Van Langendonckt A, Casanas-Roux F, Donnez J. Oxidative stress and peritoneal endometriosis. Fertil Steril 2002;77:861-70.

16. Kruse HD, Orent ER, McCollum EV. Studies on magnesium deficiency in animals: I. Symptomatology resulting from $\mathrm{Mg}$ deprivation. J Biol Chem 1932;96:519-39.

17. Haury VG, Cantarow A. Variations of serum magnesium in 52 normal and 440 pathologic patients. J Lab Clin Med 1942; 27:616-22.

18. Saris NE, Mervaala E, Karppanen H, Khawaja JA, Lewenstam A. Magnesium. An update on physiological, clinical and analytical aspects. Clin Chim Acta 2000;294:1-26.

19. Kanbay M, Goldsmith D, Uyar ME, Turgut F, Covic A. Magnesium in chronic kidney disease: challenges and opportunities. Blood Purif 2010;29:280-92. 
20. Chaumais MC, Lecerf F, Fattal S, Savale L, Günther S, Huertas A, et al. A study of magnesium deficiency in human and experimental pulmonary hypertension. Magnes Res 2012;25: 21-7.

21. Dowling O, Chatterjee PK, Gupta M, Tam Tam HB, Xue X, Lewis D, et al. Magnesium sulfate reduces bacterial LPSinduced inflammation at the maternal-fetal interface. Placenta 2012;33(5):392-8.

22. Rayssiguier Y, Mazur A. Magnesium and inflammation: lessons from animal models. Clin Calcium 2005;15:245-8.

23. Murphy AA, Santanam N, Parthasarathy S. Endometriosis: a disease of oxidative stress?. Semin Reprod Endocrinol 1998;16: 263-73.

24. Murphy AA, Santanam N, Morales AJ, Parthasarathy S. Lyso- phosphatidyl choline, a chemotactic factor for monocytes/ T-lymphocytes is elevated in endometriosis. J Clin Endocrinol Metab 1998;83(6):2110-3.

25. Campos Petean C, Ferriani RA, dos Reis RM, de Moura MD, Jordão AA Jr, Navarro PA. Lipid peroxidation and vitamin E in serum and follicular fluid of infertile women with peritoneal endometriosis submitted to controlled ovarian hyperstimulation: a pilot study. Fertil Steril 2008;90:2080-5.

26. Mier-Cabrera J, Genera-García M, De la Jara-Díaz J, PerichartPerera O, Vadillo-Ortega F, Hernández-Guerrero C. Effect of vitamins $\mathrm{C}$ and $\mathrm{E}$ supplementation on peripheral oxidative stress markers and pregnancy rate in women with endometriosis.Int J Gynaecol Obstet 2008;100:252-6.

\section{Peer Reviewers' Commentary}

Chronic pelvic pain is a common condition in women of reproductive age and can be described as chronic nociceptive, inflammatory and neuropathic pain characterized by spontaneous pain or a response to various stimuli. Oxidative stress is a component of the inflammatory reaction associated with pain processes. Iron and NF- $\kappa$ B are well-known inducers of oxidative stress, and reactive oxygen species (ROS) are associated with chronic pelvic pain and play an important role in the regulation of genes expressing immunoregulators, cytokines, and other molecules. Chronic pelvic pain treatment is often unsatisfactory and limited to symptom control. However, dietary treatment with antioxidants can improve the function of the immune system and overcome free radical damage. Therefore, dietary supplementation is suggested as a means to treat some chronic medical conditions that respond poorly to medication. In this review, dietary treatment with antioxidants could be considered for new strategy for treatment of chronic pelvic pain and may be better tolerated by patients than current treatments.

(Comment: Editorial Committee) 